\title{
ESPECIE NUEVA DEL GÉNERO HYPSELODESMUS LOOMIS (DIPLOPODA: POLYDESMIDA: CHELODESMIDAE) DEL SUROESTE DE LA REPÚBLICA DOMINICANA
}

\author{
Carlos Suriel \\ Museo Nacional de Historia Natural, Santo Domingo (MNHNSD), Calle César Nicolás Penson, Plaza de la \\ Cultura, Santo Domingo, República Dominicana. c.suriel@museohistorianatural.gov.do
}

\section{RESUMEN}

Se describe una especie nueva de milpiés del género Hypselodesmus Loomis, 1941, colectada en el suroeste de República Dominicana, isla La Hispaniola. Es la segunda especie que se registra de este género.

Palabras clave: Diplopoda, Polydesmida, Chelodesmidae, nueva especie, Hypselodesmus, La Hispaniola, Cordillera Central, República Dominicana.

\section{ABSTRACT}

Anew milliped species of the genus Hypselodesmus Loomis, 1941 is described from the southwest of the Dominican Republic, Hispaniola island. This is the second species that has been discovered for this genus.

Key words: Diplopoda, Polydesmida, Chelodesmidae, new species, Hypselodesmus, Hispaniola, Cordillera Central, Dominican Republic.

\section{INTRODUCCIÓN}

Loomis (1941) describió el género Hypselodesmus tomando como especie tipo a H. bicolor. Se basó en tres ejemplares machos y dos juveniles colectados en 1938 por P. J. Darlington en Loma Quita Espuela (1000-3000 pies de elevación, de acuerdo a su registro), República Dominicana. Tanto el holotipo como los restantes ejemplares fueron depositados en el Museo de Zoología Comparativa de la Universidad de Harvard, Estados Unidos. Desde entonces, ninguna otra especie de este género ha sido descrita, siendo considerado como monotípico endémico de La Hispaniola y reportado solo para la República Dominicana (Jeekel, 1971; Hoffman, 1979, 1999; Pérez-Asso y Pérez-Gelabert, 2001). Dos ejemplares topotipos colectados e identificados por Antonio R. Pérez-Asso están depositados en la colección de diplópodos del Museo Nacional de Historia Natural, Santo Domingo.

En la presente contribución se describe una especie nueva del género Hypselodesmus en base a ejemplares colectados en la Cordillera Central, región suroeste; de esta manera, el género pierde su condición de monotípico, aunque mantiene su endemismo para La Hispaniola.

\section{MATERIALES Y MÉTODOS}

Se estudiaron seis ejemplares adultos, cuatro machos y dos hembras, colectados durante dos expediciones realizadas a la zona de la localidad tipo en fechas (8-12)-I-2008 y (27-29)-VI-2008. Fueron depositados oficialmente en el Museo Nacional de Historia Natural, Santo Domingo (MNHNSD) junto a otros ejemplares juveniles. Se preservaron en alcohol etílico $70 \%$. La observación se realizó utilizando un microscopio estereoscópico MEIJI TECHNO modelo EMZ-5TR, con aumento hasta 45X. Los dibujos fueron realizados por el propio autor. Para dibujar las estructuras gonopodales se utilizó una cámara lúcida marca LEITZ WETZLAR. Para dibujar la silueta de los segmentos del cuerpo se utilizó como plantilla una foto del animal vivo tomada con una cámara Canon, modelo EOS 40D. Las medidas se tomaron con una Miniescala de Bio Quip Products, Inc. modelo "Métrica" con un rango de $5 \mathrm{~mm}$ y divisiones de 0.1 $\mathrm{mm}$. Las categorías taxonómicas mayores se usaron siguiendo a Hoffman (1979). Para la nomenclatura morfológica se siguió a Hoffman (1990) y Pérez-Asso (1996, 1998, 2003, 2005 a, 2005 b). La medida de longitud del cuerpo se tomó desde el extremo de la cabeza hasta el ápice del epiprocto y el ancho entre los ángulos posteriores de los paraterguitos en el segmento 7, siguiendo a Pérez-Asso (1996, Ídem). 
En cada caso y para cada ejemplar se ofrece la media de las medidas obtenidas, indicándose entre paréntesis el rango de variación y el número de ejemplares medidos. Para tomar las otras medidas se procedió como se detalla a continuación: antenas, desde el alveolo hasta el extremo del antenómero 7; patas, desde la base de la coxa hasta el extremo de la garra; ancho de la cabeza siguiendo la línea imaginaria que une las genas. Todas las medidas se expresaron en milímetros (mm). Las coordenadas geográficas y la altitud en la localidad tipo se registraron usando un Global Position Satelital (GPS) marca Garmin, modelo ETREX.

La diagnosis de la especie se elaboró estableciendo diferencias con Hypselodesmus bicolor Loomis, en base a la descripción original (Loomis, Ídem); adicionalmente se dispuso de dos ejemplares topotipos (un macho y una hembra) referidos más arriba ((MNHNSD 19.646 y 19.647). Abreviaturas: MNHNSD (Museo Nacional de Historia Natural, Santo Domingo), SX ( $\mathrm{S}=$ diplosegmento, $\mathrm{X}=$ número del diplosegmento correspondiente) y APP(ángulo posterior del paraterguito).

\title{
Clasificación.
}

\section{RESULTADOS}

\author{
Clase Diplopoda DeBlainville in Gervais, 1844 \\ Subclase Helminthomorpha Pocock, 1887 \\ Superorden Merocheta Cook, 1895 \\ Orden Polydesmida Leach, 1814 \\ Suborden Chelodesmidea Cook, 1895 \\ Superfamilia Chelodesmoidea Cook, 1895 \\ Familia Chelodesmidae Cook, 1895 \\ Subfamilia Chelodesminae Hoffman, 1979 \\ Género Hypselodesmus Loomis, 1941
}

Referencias. Hypselodesmus Loomis, 1941, Bull. Mus. Comp. Zool., 88 (2): 53-55. Jeekel, 1971, Monog. Nederl. Entom. Vereng., 5: 267. Hoffman, 1979, Mus. Hist. Nat. Genéve: 154, 236 pp; 1999, Virg. Mus. Hist. Nat., Spec. Public., 8: 288. Pérez-Asso y Pérez-Gelabert, 2001, Bol. S.E.A. 28: 74. Pérez-Gelabert, 2008, Zootaxa, 1831.530 pp.

\section{Hypselodesmus assoi sp. nov.}

(Figs.1-3, 5-7)

Diagnosis. Hypselodesmus assoi sp. nov. se diferencia de $H$. bicolor en lo siguiente: a)fémur de los gonopodos sobrepasando el proceso prefemoral y sin rodearlo completamente, solo cubriendo parcialmente su borde interno (Fig. 3); b) postfémur de ápice agudo y no bifurcado, ambas ramas se entrecruzan por arriba del proceso prefemoral (Figs. 3, 5-6); c) proceso prefemoral no subcilíndrico como en $H$. bicolor sino laminar, más estrecho en la base y con proceso dentiforme en parte media, a partir del cual se ensancha y curva hacia dentro, presentando un pliegue en su cara interna y terminando en un ápice agudo (Fig. 7); d) metaterguitos de ambos sexos castaño claro y paraterguitos ambarino claro (no blanquecinos); e) no hay diferencias de color entre segmentos poríferos y no poríferos; f) ausencia de tubérculos en metaterguitos; g) solo un diminuto proceso dentiforme en el ángulo anterior de los paraterguitos de segmentos $2-4 ; \mathrm{h}$ ) poros abriendo hacia arriba en los callos poríferos, no lateralmente; (Fig. 1), i) antenómeros 2-6 castaño rojizo.

Diagnosis. Hypselodesmus assoi sp. nov. differs from H. bicolor in the following: a) femur of gonopods exceeding the prefemoral process, around behind it but not completely, and covering partially its internal margin (Figs. 3-4); b) postfemur with acute apex, without bifurcation, entwined on the top of prefemoral process (Figs. 3, 5-6); c) prefemoral process laminar, not subcylindrical as in H. bicolor, narrower at the bottom, with a small tooth at the middle from which it widens and curves inward, with a fold in its internal face and ending in an acute apex (Fig. 7); d) metazonite of both sexes are light chestnut-brown, paranota light amber; e) poriferous and non-poriferous segments with the same color pattern; f) metazonite without tubercles; g) anterior angle of paranota with a very small tooth generally at segments $2-4$; h) pores opening upward from an oval depression, not obliquely outward; (Fig. 1), i) antennae articles 2-6 chestnut reddish. 
Descripción del Holotipo. Macho. Número catalográfico: MNHNSD19.526. Longitud=27.5 mm, Ancho $=3.8 \mathrm{~mm}$. Cuerpo espigado, con lados casi paralelos a partir de S3, no es notoriamente más ancho en los primeros cuatro segmentos. Metaterguitos castaño claro, prozonito y pleurito de tono más oscuro. Metaterguitos lisos y pulidos, excepto por una sola seta en S2, presentando una depresión central muy tenue a partir de S4 (Fig. 1). Paraterguitos ambarino claro, con diminuto proceso dentiforme, o pequeño diente definido, en el ángulo anterior de S2-S4. Presencia de poros en segmentos 5, 7, 9-10, 12-13, 15-19 (fórmula porífera normal), abriendo hacia arriba desde la excavación de un callo no pronunciado de forma oval (Fig. 1). APP con ligero levantamiento a partir de S4, inclinándose hacia atrás, en ángulo obtuso con el margen posterior del metaterguito (Fig. 1). A partir de S8 el APP se hace notoriamente agudo, en S19 se curva hacia dentro en ángulo agudo con el margen posterior del metaterguito, al tiempo que se reduce drásticamente el área del paraterguito (Fig. 2). Segmentos poríferos y no poríferos con el mismo patrón de color. Epiprocto sin modificaciones y de color ambarino claro, presenta cinco (5) pares de macrosetas apreciables en vista dorsal, dos de éstas en posición terminal (Fig. 2). Hypoprocto subtriangular, no redondeado como en $H$. bicolor; más ancho que largo y con dos macrosetas erectas. Valvas anales más largas que anchas, con estrías longitudinales y dos pares de macrosetas erectas a lo largo de su línea de unión.

Collum subelíptico, ligeramente ondulado en la porción media del borde posterior, sobrepasando lateralmente la cabeza en menos de $1 \mathrm{~mm}$, aproximadamente $0.6 \mathrm{~mm}$. Depresión en posición subcentral con 3 setas erectas, una en medio de la depresión y las dos restantes a cada lado de aquella pero no alineadas; color igual que metaterguitos (Fig. 1).

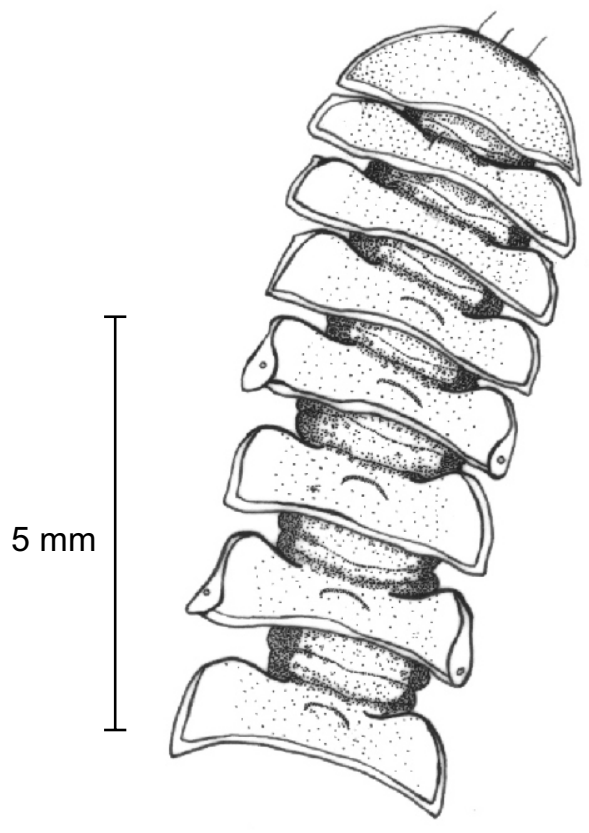

1

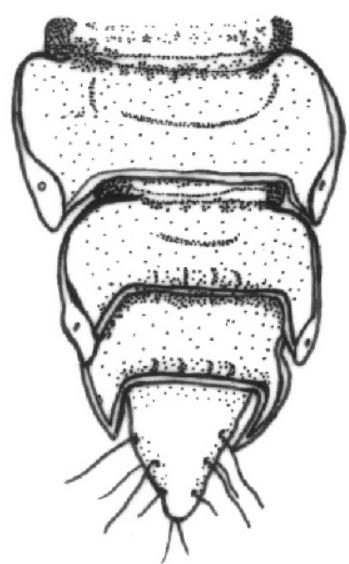

2

Figuras 1-2. Macho holotipo de H. assoi sp. nov. Vista dorsal. 1-Primeros ocho segmentos del cuerpo. 2-Segmentos 17-20, se observa el epiprocto. 
Cabeza de $2.4 \mathrm{~mm}$ de ancho. Epicraneum color castaño como metaterguitos, degradando hacia un tono más claro a partir del frons, genas aún más claras. Sutura media a lo largo del epicraneum, llegando hasta un poco antes del frons. Varios pares de setas se distribuyen a partir del epicraneum: dos pares, no alineadas, a ambos lados de la sutura media, un par de mayor tamaño flanqueando el extremo de la sutura, un poco más arriba del frons; otro par de macrosetas más abajo, cada una junto al alveolo antenal correspondiente y otras dispersas en la región del clypeus. Antenas largas $(5.1 \mathrm{~mm})$, alcanzando el APP en S4 cuando se extiende hacia atrás. Antenómeros pubescentes, 2-6 aproximadamente del mismo tamaño y grosor, de color castaño rojizo, excepto 1 y 7 que son blanquecinos, siendo el 6 de tono más acentuado. Distancia entre las bases de las antenas $0.8 \mathrm{~mm}$.

Esternitos del mismo color que paraterguitos, pubescentes, presentando mayor número de setas al nivel del par anterior de patas (20-22), en el par posterior se cuentan 7-10. Patas largas, sobresaliendo del cuerpo apreciablemente, $4.2 \mathrm{~mm}$ de longitud medidas en el par posterior de S8. Podómeros pubescentes, principalmente el tercero (fémur) y el sexto (tarso).

Gonopodos largos, alcanzando el par posterior de patas de S6. Conjunto del fémur y postfémur acintado, curvado en forma de "s", con la curvatura inferior mucho más larga y hacia adentro, la curvatura superior hacia fuera (Fig. 3). Longitud desde la coxa hasta el inicio de la curvatura inferior $1.5 \mathrm{~mm}$. El fémur termina en un pequeño diente; a partir de este pequeño diente se inicia el postfémur, estrecho y lanceolado, curvando hacia fuera, con ápice agudo y entrecruzándose (los de ambas ramas) por arriba del proceso prefemoral (Figs. 3, 5-6). Longitud desde el diente hasta el inicio de la flexión del postfémur 0.3 $\mathrm{mm}$. El proceso prefemoral es laminar, más estrecho en la base, con un proceso dentiforme en su porción media apuntando hacia dentro; a partir de este proceso se ensancha aún más y se curva hacia dentro, presentando un pliegue en su cara interna, su ápice es agudo; se extiende casi hasta el nivel del postfémur (Figs. 5 y 7). La porción curvada del fémur cubre parcialmente el borde interno del proceso prefemoral, sobresaliendo de éste el proceso dentiforme de su parte media (Fig. 3). Modificaciones sexuales pregonopodales consistentes en una estructura cilíndrica corta, delgada, que se levanta sobre cada coxa en el segundo par de patas.

Variabilidad. Machos: longitud=26,7 $\mathrm{mm}(25,5-27,5 ; \mathrm{n}=4)$, anchura=3,8 $\mathrm{mm}(3,7-4,0 ; \mathrm{n}=4)$. Hembras: longitud=30,5 mm (30,0-31,0; $\mathrm{n}=2)$, anchura=4,35 mm (4,2-4,5; $\mathrm{n}=2)$. Anchura en S3: machos, 4,05 mm $(3,9-4,2 ; n=4)$; hembras, $4,75 \mathrm{~mm}(4,7-4,8 ; n=2)$. La distancia entre las antenas no presentó variabilidad entre los ejemplares de un mismo sexo: $0,8 \mathrm{~mm}$ en los machos y $1,0 \mathrm{~mm}$ en las hembras. Anchura de la cabeza: machos, 2,4 mm (2,3-2,5; $\mathrm{n}=4)$; hembras, 2,8 $\mathrm{mm}(2,8-2,8 ; \mathrm{n}=2)$. El dentículo, a veces solo como un proceso dentiforme, se presenta invariablemente en segmentos 2-4; reaparece con poca definición en S6 del ejemplar 19.109, en S8 de 19.110, en solo uno de los dos paraterguitos de S5 en ejemplar 19.112 y en S5-S6 del 19.526; no reaparece en otros segmentos de 19.111 y 19.113.

Etimología. El epíteto específico hace honor al zoólogo Antonio R. Pérez-Asso, gran conocedor y consagrado estudioso de los milpiés a quien el autor debe su iniciación y actual realización en tan placentero como inusual quehacer.

Comentario. La descripción del género Hypselodesmus por Loomis (1941) se hizo disponiendo solo de la especie tipo, Hypselodesmus bicolor, razón por la cual no era posible separar las características genéricas de las propiamente específicas. La descripción de $H$. assoi sp. nov. sugiere la necesidad de una redescripción posterior del género. Por el momento, basándonos en la descripción original de H. bicolor, en el examen de topotipos de esta especie y en el estudio de los ejemplares de $H$. assoi sp. nov, consideramos como el rasgo genérico más distintivo la presencia de gonopodos con fémur acintado, flexionado en forma de "s" abrazando el proceso prefemoral, con postfémur igualmente acintado y curvado hacia fuera, características inconfundibles con especie alguna en la fauna de diplópodos de La Hispaniola.

Tipos. Holotipo: macho MNHNSD 19.526. Paratipos: machos MNHNSD 19.109- 19.110 y 19.111; hembras, MNHNSD 19.112 y 19.113. Todos estos especímenes fueron depositados en el MNHNSD. Colectados en Sabana Vieja, Provincia San Juan, República Dominicana; en área del Parque Nacional José del Carmen Ramírez, Cordillera Central, N 1904' 45", W 071 11' 52"; altitud: 1,944 m., entre pajones e hierbas del sotobosque de un pinar y bajo corteza de un pino seco a $0,5 \mathrm{~m}$ de altura. Colectores: Rosa Rodríguez, Gabriel de los Santos, Alexander Sánchez-Ruiz, Elvi de los Santos, Miguel Ángel Landestoy y Carlos Suriel; 10-I-2008, 9:00-12:00 meridiano, nublado; 28-VI-2008, 3:00-4:30 pm, bajo lluvia. Diplópodos asociados en el lugar de colecta: Stemmiulidae y Pyrgodesmidae.

Distribución geográfica. Hasta ahora solo conocida de la localidad tipo. 


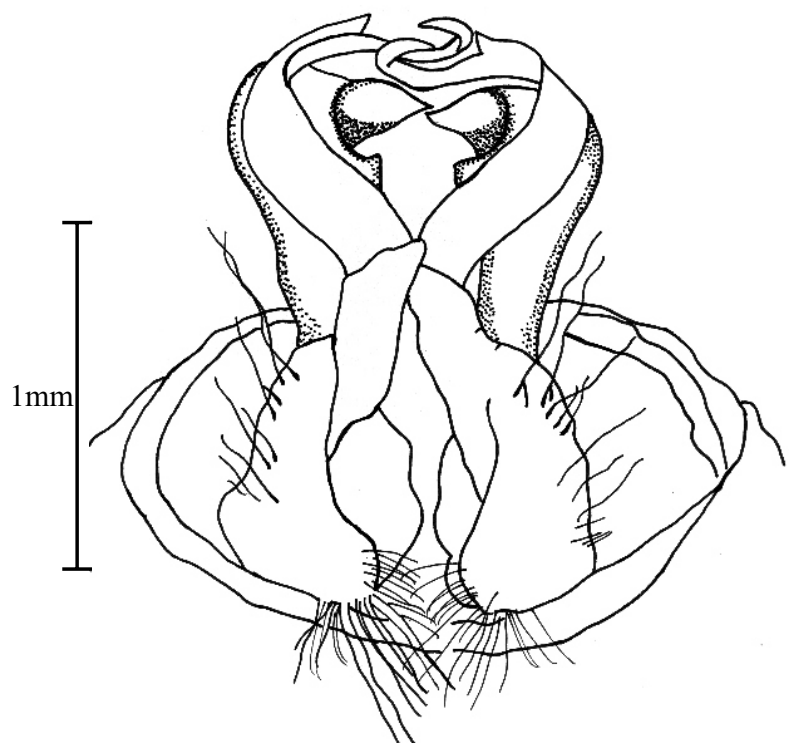

3

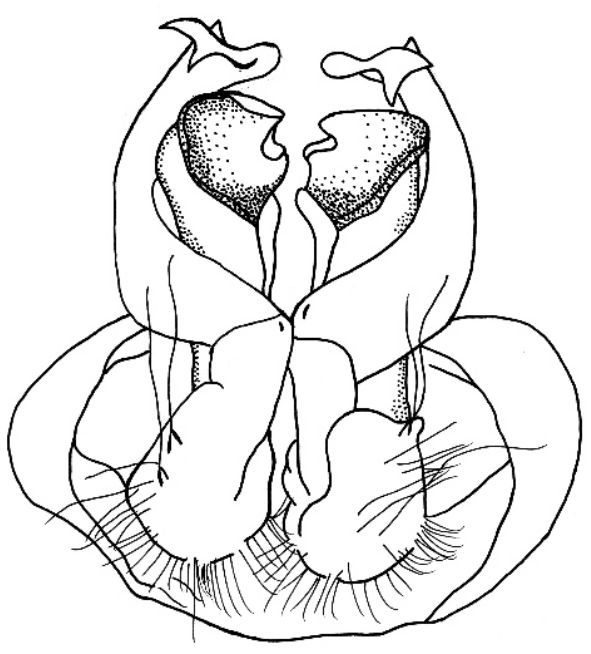

4
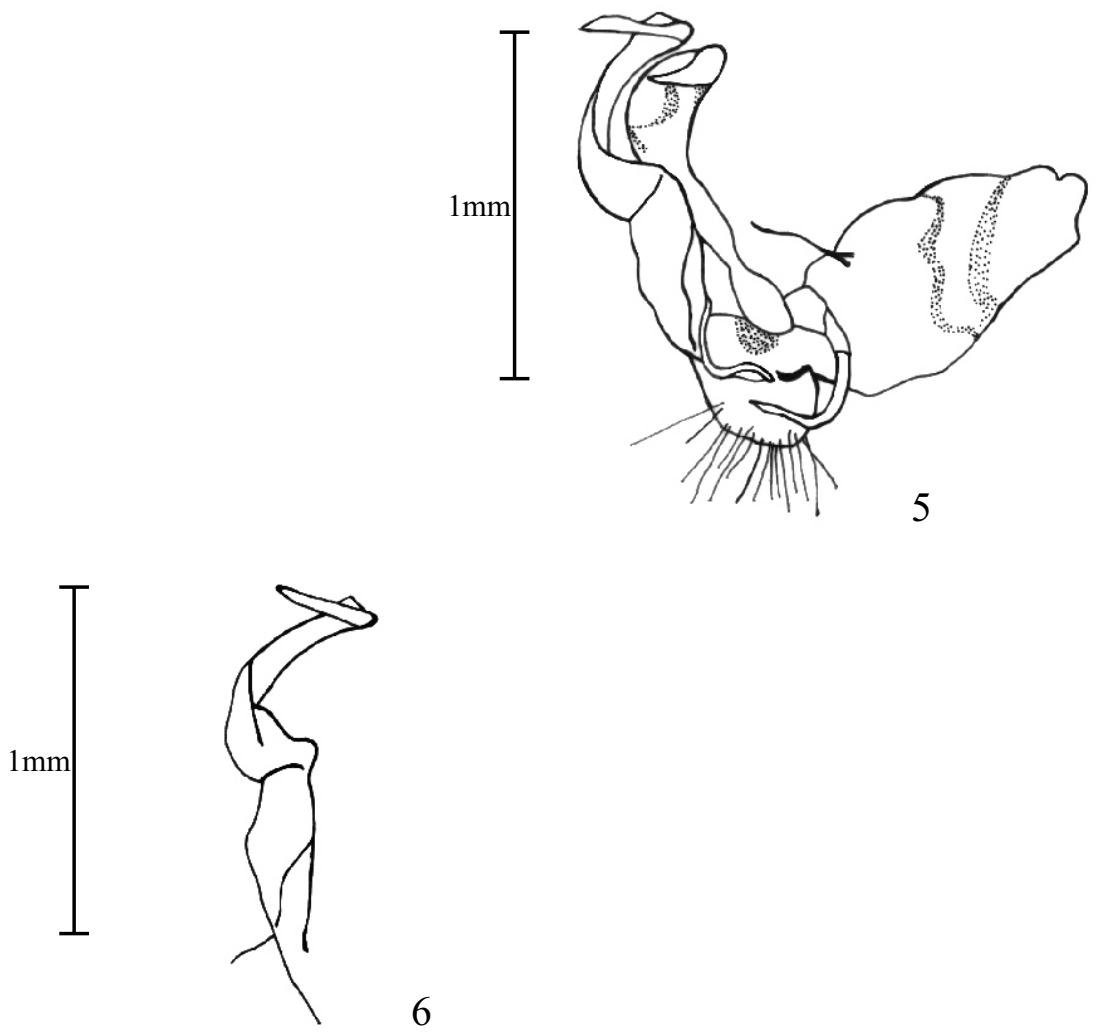

6

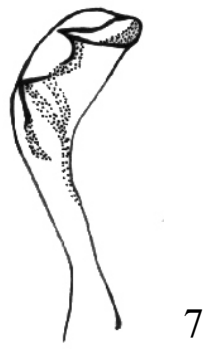

Figuras 3-7. Macho de H. assoi sp. nov., excepto fig. 4 (topotipo de H. bicolor). 3-Gonopodos in situ holotipo de H. assoi sp. nov. en vista posterior. 4- Gonopodos in situ topotipo de $H$. bicolor en vista posterior. 5-Rama de gonopodos (extraída) de $H$. assoi sp. nov. en vista anterolateral. 6-Fémur extraído de $H$. assoi sp. nov. en vista anterior. 7-Proceso prefemoral extraído de $H$. assoi sp. nov. en vista anterior. 


\section{AGRADECIMIENTOS}

Antonio R. Pérez-Asso hizo correcciones y sugerencias pertinentes en base al examen de los especímenes y la lectura del manuscrito. Julio A. Genaro hizo sugerencias que permitieron mejorar la redacción del manuscrito. Celeste Mir, Directora del Museo Nacional de Historia Natural (MNHNSD), realizó correcciones a la redacción en inglés del Resumen (Abstract) y la Diagnosis. El Consorcio Ambiental Dominicano (CAD), en la persona de Sésar Rodríguez, financió una de las dos expediciones. Rosa Rodríguez, Gabriel de los Santos, Alexander Sánchez-Ruiz, Elvi de los Santos y Miguel A. Landestoy ofrecieron valiosa ayuda en las colectas. Luís Gómez Sipión, de la Dirección de Áreas Protegidas (SEMARENA) ayudó en la organización y logística de las expediciones. Julián, Sandro y Rafael Sánchez asistieron como guías. A todos ellos, nuestro agradecimiento.

\section{LITERATURA CITADA}

Loomis, H. F. 1941. Millipeds collected in Puerto Rico and the Dominican Republic by Dr. P. J. Darlington in 1938. Bull. Mus. Comp. Zool., 88 (2): 53-55. Fig. 19.

Jeekel, C. A. W. 1971. Nomenclator generum et familiarum Diplopodorum: A list of the genus and family-group names in the Class Diplopoda from the $10^{\text {th }}$ edition of Linnaeus 1758, to the end of 1957. Nederl. Ent. Ver., Amsterdam, 5: 267.

Hoffman, R. L. 1979. Clasification of the Diplopoda. Mem. Mus. His. Nat. Geneve. 237 pp.

Hoffman, R. L. 1990. Diplopoda. 835-860. En : D. L. Dindall, et al., Soil Biology Guide. Wiley Interscience, New York. 1,349 pp.

Hoffman, R. L. 1999. Checklist of the millipedes of North and Middle America. Virg. Mus. Nat. Hist. Spec. Public., 8: 288 pp.

Pérez-Asso, A. R. 1996. Revisión del género Amphelictogon (Diplopoda: Polydesmida: Chelodesmidae) en Cuba. Insecta Mundi, 10: (1-4), 181-216.

Pérez-Asso, A. R. 1998. Nuevas especies del género Amphelictogon (Diplopoda: Polydesmida: Chelodesmidae) en Cuba. Insecta Mundi, 12 (3-4): 161-173.

Pérez-Asso, A. R. 2003. Nuevo género y especie de milpiés de la familia Chelodesmidae (Diplopoda: Polydesmida) para La Hispaniola. Solenodon, 3: 57-60.

Pérez-Asso, A. R. 2005 a. Dos especies y una subespecie nueva de milpiés del género Achromoporus (Diplopoda: Polydesmida) para Hispaniola. Solenodon, 5: 53-59.

Pérez-Asso, A. R. 2005 b. Nuevo género y especie de milpiés de la familia Chelodesmidae (Diplopoda: Polydesmida) para Hispaniola. Solenodon, 5: 60-63.

Pérez-Asso, A. R. y D. E. Pérez-Gelabert. 2001. Checklist of the millipeds (Diplopoda) of Hispaniola. Boletín S.E.A. 28: 67-80.

Pérez- Gelabert, D. E. 2008. Arthropods of Hispaniola (Dominican Republic and Haiti): A checklist and bibliography. Zootaxa 1831. 1-530. Magnolia Press, Auckland, New Zealand. Online edition. 